\title{
Insecticide susceptibility status of human biting mosquitoes in Muheza, Tanzania
}

\author{
BASILIANA EMIDI ${ }^{1,2^{*}}$, WILLIAM N. KISINZA ${ }^{3}$, ROBERT D. KAAYA ${ }^{1}$, ROBERT MALIMA ${ }^{3} \&$ FRANKLIN W. MOSHA \\ ${ }^{1}$ Kilimanjaro Christian Medical University College, P.O. Box 2240, Moshi, Tanzania \\ ${ }^{2}$ National Institute for Medical Research, Headquarters, P.O. Box 9653, Dar es Salaam, Tanzania \\ ${ }^{3}$ National Institute for Medical Research, Amani Research Centre, P.O. Box 81, Muheza, Tanzania
}

\begin{abstract}
Background: There has been a rapid emergence in insecticide resistance among mosquito population to commonly used public health insecticides. This situation presents a challenge to chemicals that are currently used to control mosquitoes in sub-Saharan African. Furthermore, there is limited information on insecticide susceptibility status of human-biting mosquitoes in some areas of Tanzania. This study aimed to determine insecticide susceptibility status of human biting mosquitoes in a rural area of north-eastern Tanzania.

Methods: The study was conducted in two villages in Muheza district, Tanzania. Insecticide susceptibility bioassays were performed according to the World Health Organization standard operating procedures on two to five-day old human biting mosquitoes. The mosquitoes of each species were exposed to four classes of insecticides commonly used for malaria vector control. Mosquito mortality rates (\%) were determined after 24 hours post insecticide exposure.

Results: Mosquito species tested were Anopheles gambiae s.l., An. funestus, Aedes aegypti, and Culex quinquefasciatus species. Real-time PCR have showed that the main sibling species of An. gambiae complex and An. funestus group were An. gambiae s. s. (58.2\%) and An. funestus s. s. (91.1\%), respectively. All mosquitoes, except Ae. aegypti formosus were susceptible to pirimiphos-methyl (0.25\%). An. gambiae s. I. was found to be resistant to permethrin (0.75\%) but showed possibility of resistance to DDT (4\%) and bendiocarb (0.1\%). Our findings have shown that, An. funestus was fully susceptible to all insecticide tested.

Conclusion: The present study has revealed different levels of insecticide susceptibility status to four classes of commonly used insecticides in the most common mosquito vectors of human diseases in north-eastern Tanzania. The findings of the present study call for integrated vector control interventions.

Keywords: insecticide resistance, mosquitoes, Tanzania
\end{abstract}

\section{Introduction}

Mosquito-borne diseases such as dengue, Rift Valley fever, malaria and lymphatic filariasis remain the major cause of illness and deaths in many parts of the world, particularly in tropical and sub-tropical climates (Goddard, 2008). Chemical insecticides remain the main tool for mosquito control although it is challenged by the development of resistance to commonly used public health insecticides (Gatton et al., 2013; Nkya et al., 2013; David et al., 2013). The emergence and rapid spread of pyrethroid resistance in Anopheles gambiae complex populations may be a threat for the sustainability of malaria control programme for both indoors residual spray (IRS) and insecticide treated mosquito nets (ITNs) (Ranson et al., 2011). Resistance has developed to common classes of insecticidal active ingredients used for mosquito vector control, mainly the synthetic-derived pyrethroids (Chareonviriyaphap et al., 2013). Resistance to malathion, fenitrothion, propoxur, DDT and chlorpyriphos in Culex quinquefasciatus has been reported in Brazil (González et al., 1999; Bracco et al., 1999). The occurrence of insecticide resistant Culex mosquitoes has also been reported in Wete, Tanzania against DDT, deltamethrin, permethrin and lambda-cyhalothrin (Jones et al.,2012). In Northwestern Tanzania, An. gambiae s.s. has been reported to be resistant to bendiocarb (Gatton et al.,

\footnotetext{
${ }^{*}$ Correspondence E-mail: ebasi2002@gmail.com
} 
2013; Protopopoff et al., 2013; Matowo et al., 2015). The use of agricultural pesticides, ITNs and IRS for malaria vector control and the use of acaricides against pests of veterinary importance, is strongly suspected to be responsible for the emergence of resistance in Tanzania (Nkya et al., 2014).

In Tanzania, insecticide resistance until recently was mainly reported in An. gambiae s.l. (Kabula et al., 2012, 2013, 2014; Protopopoff et al., 2013; Nkya et al., 2014; Matowo et al., 2010, 2014, 2015) while there is only one report of insecticide resistance of DDT, bendiocarb and pyrethroids such as permethrin, deltamentrin and lambdacyhalothrin in An. funestus in the country (Lwetoijera et al., 2014). There is no report in Tanzania of insecticide susceptibility status in An. funestus to Pirimiphosmethyl insecticide. Furthermore, there is no report regarding insecticides susceptibility to four classes of insecticides in other human biting mosquitoes in Tanzania including Cx. quinquefasciatus, and Ae. Aegypti. Therefore, the present study aimed to determine the insecticide susceptibility status of four species of human biting mosquitoes to four classes of commonly used insecticides in northeastern Tanzania.

\section{Material and Methods}

\section{Study site}

This cross-sectional study was conducted between May 2015 and March, 2016 in Muheza District of north-eastern Tanzania. The district covers a geographical area of $4922 \mathrm{~km}^{2}$, stretching from a coastal plain at sea level to the East Usambara Mountains at 1,050 $\mathrm{m}$ above sea level. The climate is tropical, with dense rainforest over the Usambara mountain ranges with annual rainfall $1000-2000 \mathrm{~mm}$. According to the 2012 Population and Housing census, the district has a total of 204,461 people (URT, 2013). The area is endemic for malaria and lymphatic filariasis (Simonsen et al., 2014; Derua et al., 2015). Two villages, namely Zeneti ( $\left.5^{\circ} 13^{\prime} 23.44^{\prime \prime S} ; 38^{\circ} 39^{\prime} 32.6^{\prime \prime E}\right)$ and Mkuzi (5 $5^{\circ} 3^{\prime} 49.9^{\prime \prime S}$; $\left.38^{\circ} 50^{\prime} 19 \cdot 44^{\prime \prime E}\right)$ were selected for mosquito sampling.

\section{Mosquito collection and identification}

Mosquito larvae were collected from the wild in order to obtain an age-standardized sample of the adult population for the insecticide bioassay tests. Mosquito larvae were collected by using a standard dipper in a variety of breeding sites, including rice paddy, rain water collection pools, road potholes, hoof prints around swamps at Zeneti and Mkuzi villages. Collected larvae were sorted to remove larval predators. Culicine and Anopheline larvae were kept in separate larval rearing containers basing on their larval stages. Emerged pupae were sucked from the larval containers using a plastic pipette and placed in plastic cups inside the mosquito cages to prevent emerging adult mosquitoes from escaping. Emerged adult mosquitoes were identified morphologically using identification keys (Edwards, 1941; Gillies \& De Meillon, 1968; Huang, 2004). An. gambiae s.l. and An. funestus were further identified to sibling species by Real-Time Polymerase Chain Reaction (PCR) (Bass et al., 2007).

\section{Insecticide susceptibility bioassays}

Insecticide susceptibility bioassays were performed according to standard guidelines (WHO, 2013). Assays were carried out with four insecticides namely, DDT ( $4 \%$ ), permethrin ( $0.75 \%$ ), bendiocarb (0.1\%) and pirimiphos-methyl (0.25\%). Each type of insecticides was replicated five times. Of the five replicates, one was a control replicate. Twenty to 25 mosquitoes per tube were hence, a sample size of 100 to 125 mosquitoes was tested for each insecticide. Two to five-day old female mosquitoes were used for bioassay tests. Tested mosquitoes were monitored at different time interval of 10, 15, 20, 30, 40, 50 and 60 minutes. An. gambiae s. I., An. funestus, Ae. aegypti formosus and Cx. 
quinquefasciatus mosquitoes were tested against the four (4) insecticides belonging to the major public health insecticide classes.

In the tested mosquitoes, the numbers knocked-down were recorded. Cx. quinquefasciatus was exposed for 80 minutes because knockdown was below $80 \%$ after the 60 -minute exposure to Permethrin (0.75\%) and DDT (4\%). After exposure time, all mosquitoes were transferred to the holding tubes and provided with $10 \%$ glucose solution through a cotton wool. The mortality rates were determined at 24 hours post exposure. In each bioassay, a control experiment using papers impregnated only with insecticide carrier oil, was performed in the same way as in treatment experiments. Susceptibility tests were conducted in the laboratory under $25 \pm 2{ }^{\circ} \mathrm{C}$ and $70-80 \%$ temperature and humidity, respectively. Dead and surviving mosquitoes at the end of an experiment were kept in separate Eppendorf tubes containing silica gel and labelled (WHO, 2013; Umar et al., 2014).

\section{Data analysis}

The knockdown (KD) mosquito data was subjected to Polo Plus probit and logit analysis version 1, 2002-2009 LeOra Software to estimate the KDT50 and KDT95, which is the time taken to knock down $50 \%$ and $95 \%$ of the exposed mosquitoes, as well as their $95 \%$ confidence interval. The $24 \mathrm{hr}$ mortality rate (\%) was established by counting the number of mosquitoes killed at the end of the holding period ( 24 hours) divided by the total number of mosquito exposed times 100 . The insecticide susceptibility status of tested mosquitoes was assessed based on standard guidelines (WHO, 2013). Mortality range $98-100 \%$ indicates susceptible mosquito population, $90-97 \%$ suggests possible resistance that need to be confirmed and below $90 \%$ indicates existence of resistance. The adult mosquito mortalities in control experiments were less than $5 \%$, therefore no correction made by Abbott's formula (WHO, 2013).

\section{Ethical considerations}

The present study was approved by the Medical Research Coordinating Committee (NIMR/HQ/R.8a/Vol.IX/1613) and Kilimanjaro Christian Medical University College Ethics committee (Ethical Clearance No.885).

\section{Results}

\section{Mosquito composition}

A total of 2,515 mosquitoes comprising 1520 (60.4\%) from Zeneti and 995 (39.6\%) from Mkuzi village were tested to four classes of insecticides in order to assess their susceptibility status. A total of 528 Anopheles mosquitoes were identified by real-time PCR. Among these, An. gambiae s.l. accounted for 91.5\% ( $n=483)$ and An. funestus group was 8.5\% $(n=45)$. An. gambiae s.l. was composed of An. gambiae s.s. (58.2\%) and An. arabiensis (41.8\%). Within the An. funestus group, four sibling species were identified which were An. funestus s.s. (91.1\%), An. rivulorum (4.4\%), An. leesoni and An. parensis (Table 1). The none-amplified and the missing samples were excluded in the analysis.

\section{Mosquito mortality rate}

The $24 \mathrm{hr}$ mortality rate post-exposure to insecticides revealed resistant of An. gambiae s.l. to permethrin (0.75\%) and bendiocarb (0.1\%) with mortalities of $72 \%$ and $87 \%$, respectively for mosquitoes collected from Zeneti village. In this village, An. gambiae s.l. was susceptible to DDT (4\%) and pirimiphos-methyl (0.25\%). For mosquitoes collected from Mkuzi village, An. gambiae s.l. was also resistant to permethrin (0.75\%) with mortality rate of $69 \%$. However, with exposure to DDT (4\%), An. gambiae s.l. exhibited possible resistance. Unlike, Zeneti village, An. gambiae s.l. from 
Mkuzi village was susceptible to bendiocarb (0.1\%) and pirimiphos-methyl (0.25\%). An. funestus from Zeneti village were susceptible to all insecticides tested (permethrin, DDT, bendiocarb, pirimiphosmethyl (Table 2).

Table 1: An. gambiae complex and An. funestus group sibling species identification

\begin{tabular}{llll}
\hline Species complex & Sibling Species & Total (n) & $\%$ \\
\hline An. gambiae s.l. & An. arabiensis & 202 & 38.3 \\
An. funestus & An. gambiae s.s. & 281 & 53.2 \\
& An. funestus s.s. & 41 & 7.8 \\
& An. leesoni & 1 & 0.2 \\
Total & An. parensis & 1 & 0.2 \\
\hline
\end{tabular}

In both villages, $C x$. quinquefasciatus was resistant to three classes of insecticides tested except for pirimiphos-methyl (0.25\%). Ae. aegypti formosus was susceptible to all insecticides tested at Mkuzi village. Ae. aegypti formosus collected from Zeneti exhibited possible resistance to DDT (4\%) and pirimiphos-methyl (0.25\%).

Table 2: Insecticide resistance status of mosquito species exposed to four classes of insecticides in two villages

\begin{tabular}{|c|c|c|c|c|c|c|c|c|c|}
\hline \multirow{2}{*}{$\begin{array}{l}\text { Study } \\
\text { Site }\end{array}$} & \multirow[t]{2}{*}{ Species } & \multicolumn{2}{|c|}{ Permethrin } & \multicolumn{2}{|l|}{ DDT } & \multicolumn{2}{|c|}{ Bendiocarb } & \multicolumn{2}{|c|}{ Pirimiphos methyl } \\
\hline & & \%Mortality & $\mathbf{R S}^{\mathbf{a}}$ & \%Mortality & $\mathbf{R S}^{\mathbf{a}}$ & \%Mortality & $\mathrm{RS}^{\mathrm{a}}$ & \%Mortality & $\mathrm{RS}^{\mathrm{a}}$ \\
\hline \multirow[t]{4}{*}{ Zeneti } & An. gambiae s.l. & 72 & $\mathrm{R}$ & 99 & $S$ & 87 & $\mathrm{R}$ & 99 & $S$ \\
\hline & An. funestus & 100 & $\mathrm{~S}$ & 100 & $S$ & 100 & $\mathrm{~S}$ & 100 & $S$ \\
\hline & Cx. quinquefasciatus & 12 & $\mathrm{R}$ & 56 & $\mathrm{R}$ & 9 & $\mathrm{R}$ & 100 & $S$ \\
\hline & Ae. aegypti formosus & 100 & $\mathrm{~S}$ & 96 & PR & 100 & $S$ & 94 & PR \\
\hline \multirow[t]{3}{*}{ Mkuzi } & An. gambiae s.l. & 69 & $\mathrm{R}$ & 96 & PR & 100 & $\mathrm{~S}$ & 99 & $\mathrm{~S}$ \\
\hline & Cx. quinquefasciatus & 17 & $\mathrm{R}$ & 60 & $\mathrm{R}$ & 26 & $\mathrm{R}$ & 100 & $S$ \\
\hline & Ae. aegypti formosus & 100 & $\mathrm{~S}$ & 100 & S & 100 & $S$ & 100 & $S$ \\
\hline
\end{tabular}

Key: ${ }^{a} \mathrm{WHO}$ criteria for assessing susceptibility to insecticides of mosquitoes; RS=Resistance status; R=resistance, $\mathrm{S}=$ susceptible and $\mathrm{PR}=$ Possible resistance

\section{Mosquito knockdown times}

DDT (4\%) and permethrin (0.75\%) were able to knock down $50 \%$ of most of the mosquito species (KDT50) exposed within 1 hour. However, the KDT50 for Cx. quinquefasciatus was 156.5 minutes when exposed to permethrin (0.75\%). The mean-time required to knock down $50 \%$ of the mosquitoes exposed to permethrin (0.75\%) ranged from 16.8 to 27.7 minutes for Ae. aegypti formosus and 17.9 to 156.5 minutes for $C x$. quinquefasciatus (Table 3). 
Table 3: Knockdown times (KDTs) of mosquitoes exposed to DDT and Permethrin by site

\begin{tabular}{|c|c|c|c|c|c|}
\hline Site & Species & $\begin{array}{l}\text { Insecticide } \\
\text { (\%concentration) }\end{array}$ & $n^{b}$ & $\mathrm{KDT}_{50}{ }^{c}(95 \% \mathrm{Cl})$ & $\mathrm{KDT}_{95}^{\mathrm{d}}(95 \% \mathrm{Cl})$ \\
\hline \multirow[t]{8}{*}{ Zeneti } & An. gambiae s. I. & Permethrin (0.75\%) & 100 & $45.8(43.0-48.6)$ & $60.0(55.5-69.1)$ \\
\hline & & DDT (4\%) & 100 & $37.7(31.7-42.5)$ & $52.5(45.8-74.7)$ \\
\hline & An. funestus & Permethrin (0.75\%) & 100 & $30.5(27.7-33.5)$ & $68.6(58.7-85.7)$ \\
\hline & & DDT (4\%) & 100 & $35.8(34.4-37.1)$ & $51.2(48.6-54.9)$ \\
\hline & Cx. quinquefasciatus & Permethrin (0.75\%) & 100 & $156.5(114.4-378.1)$ & $393.9(214.4-2295.8)$ \\
\hline & & DDT (4\%) & 100 & $* \mathrm{e}$ & $* \mathrm{e}$ \\
\hline & Ae. aegypti formosus & Permethrin (0.75\%) & 80 & $16.8(15.4-18.2)$ & $28.4(25.2-34.1)$ \\
\hline & & $\operatorname{DDT}(4 \%)$ & 80 & $56.4(53.5-59.8)$ & $104.6(93.4-122.4)$ \\
\hline \multirow[t]{6}{*}{ Mkuzi } & An. gambiae s. I. & Permethrin $(0.75 \%)$ & 80 & $43.2(36.3-54.8)$ & $187.4(119.5-437.1)$ \\
\hline & & DDT $(4 \%)$ & 80 & $37.5(35.8-39.2)$ & $59.1(55.1-64.9)$ \\
\hline & Cx. quinquefasciatus & Permethrin (0.75\%) & 89 & $17.9(12.2-23.7)$ & $36.1(26.7-86.0)$ \\
\hline & & DDT (4\%) & 80 & $56.2(52.8-60.1)$ & $69.5(63.9-84.0)$ \\
\hline & Ae. aegypti formosus & Permethrin (0.75\%) & 80 & $27.7(25.5-29.9)$ & $47.6(42.8-55.2)$ \\
\hline & & DDT $(4 \%)$ & 83 & $44.3(42.3-46.3)$ & $74.6(69.0-82.5)$ \\
\hline
\end{tabular}

Note: $\mathrm{n}^{\mathrm{b}}=$ total number of mosquitoes exposed to each insecticide, $\mathrm{KDT}_{50} \mathrm{c}=$ time $(\mathrm{min})$ required to knock down $50 \%$ of the mosquitoes exposed to an insecticide, $\mathrm{KDT} 5^{\mathrm{d}}=$ means time $(\mathrm{min})$ required to knock down $95 \%$ of the mosquitoes exposed to an insecticide, $95 \% \mathrm{Cl}=95 \%$ Confidence Interval, *e $=$ the estimates of KDT50, KDT95 and their associated $95 \%$ Confidence Interval could not be estimated by the Probit model.

\section{Discussion}

The present study has demonstrated the occurrence of mosquito resistance to important mosquito species in north-eastern Tanzania. Our findings are consistent with other previous surveys conducted in the area (Kisinza et al., 2013) and elsewhere in Tanzania (Kabula et al., 2014; Matowo et al., 2014). Various drivers of insecticide resistance in An. gambiae s.l. in Tanzania have been identified to include the use of insecticide-treated nets and agricultural pesticides (Nkya et al., 2013). Various countries have also reported permethrin resistance in An. gambiae s.l., including Benin, Gambia and Nigeria (Yadouleton et al., 2010; Betson et al., 2013; Umar et al., 2014).

In Mkuzi village, An. gambiae s.l. showed possible resistance to DDT (4\%). This finding is similar to reports from previous results in Muheza (Kisinza et al., 2013). An. gambiae s.l. from Zeneti were susceptible to DDT (4\%). This is in contrary to previous findings in Tanzania (Kabula et al., 2012, 2013; Kisinza et al., 2013) and Benin (Yadouleton et al., 2010) which reported the species to be resistant to DDT (4\%). This is likely to be due to use of agricultural pesticides in the area (Nkya et al., 2013, 2014; Philbert et al., 2014; Reid \& McKenzie, 2016).

In the present study, An. gambiae s.l. collected from Zeneti were found to be resistant to bendiocarb (0.1\%). However, previous studies in Muheza district have shown that the species was susceptible to bendiocarb (0.1\%) (Kisinza et al., 2013; Nkya et al., 2014). These findings suggest that, insecticide resistance is rapidly increasing and its occurrence within the same locality may be different due to various environmental factors (Nkya et al., 2013; Philbert et al., 2014). An. gambiae s.I. from Mkuzi were susceptible to bendiocarb (0.1\%). Similar findings have been reported by previous results in the same area (Kisinza et al., 2013; Nkya et al., 2014). However, studies elsewhere in Tanzania and Benin have reported that An. gambiae s.l. mosquitoes are resistant to bendiocarb (0.1\%) (Matowo et al., 2015; Aïkpon et al., 2013).

In this study, An. gambiae s.l. was found to be susceptible to pirimiphos-methyl. Our findings concur with results from previous studies in Muleba, Tanzania (Matowo et al., 2015) and Atacora, 
Benin (Aïkpon et al., 2013). Pirimiphos-methyl formulation has been reported in Zambia and Zanzibar to be highly effective and appropriate insecticide for IRS and can be used for management of insecticide resistance in malaria vectors (Chanda et al., 2013; Haji et al., 2015).

The present study has shown that, An. funestus is susceptible to all insecticide tested. An. funestus is an important vector of malaria and lymphatic filariasis in north-eastern Tanzania (Derua et al., 2015). This is the first study in Tanzania to assess insecticide susceptibility status of An. funestus on pirimiphos-methyl. The susceptibility to insecticide status of An. funestus implies that, the existing common classes of insecticides used for public health can still be used for control of An. funestus. However, a study in south-eastern Tanzania has reported An. funestus to be highly resistant to permethrin (0.25\%), bendiocarb (0.1\%) and DDT (4\%) (Lwetoijera et al., 2014). The underlying reasons for the differences between the findings of these studies could be due to environmental factors including differences in agricultural activities.

The occurrence of insecticide resistance in mosquitoes is dynamic and sometimes focal. Our findings are consistent with previous studies on the An. funestus to organophosphates (malathion and fenitrothion) with 100\% mortality (Riveron et al., 2015), pirimipho-methyl (0.25\%) and DDT (4\%) (Choi et al., 2014). In previous studies in Malawi and Kwazulu-Natal, South Africa An. funestus showed high resistance to pyrethroid insecticides (Riveron et al., 2015; Hargreaves et al., 2000). In Kwazulu-Natal, An. funestus resistance to deltamethrin used for IRS, led to an increase in malaria incidence to over six-folds in during late 1990 s (Hargreaves et al., 2000). An. funestus resistance to DDT and bendiocarb has been reported in Malawi, Zambia and Zimbabwe (Choi et al., 2014; Riveron et al., 2015).

Our findings have shown that, $C x$. quinquefasciatus is highly resistant against majority of classes of insecticides tested. Multiple resistances to deltamethrin, permethrin, malathion, fenitrothion, propoxur, DDT, chlorpyriphos and lambda-cyhalothrin have been reported in $C_{x}$. quinquefasciatus in Brazil (González et al., 1999; Bracco et al., 1999), Wete Island in Tanzania (Jones et al.,2012) and India (Karlekar et al., 2013). In a situation where resistance is very high, other control measures such as environmental management such source reduction and selection of effective compounds can be opted for successful mosquito control. The present study has found that, $C x$. quinquefasciatus was fully susceptible to pirimiphos-methyl. Our findings concur with those of Ansaria et al. (2004) in India and Rowland et al. (2013) in Benin.

Interestingly, our study has documented the existence of Ae. aegypti formosus a sub-species of Ae. aegypti for the first time in the study area. Aedes aegypti consists of two sub-species namely Ae. aegypti aegypti and Ae. aegypti formosus. Ae. aegypti formosus showed possible resistance to pirimiphos-methyl. This insecticide is used for agricultural purposes in Tanzania. Our findings concur with those of a study in Brazil where Ae. aegypti was reported to be resistant to temephos which is an organophosphate (Lima et al., 2011; Macoris et al., 2014). Temephos resistance may occur due to alterations in the target site of the insecticide; the acetylcholinesterase gene or through elevated levels or differential efficacy of metabolic genes (Lima et al., 2011). Additionally, Ae. aegypti formosus exhibited possible resistance to DDT. The findings of the present study are supported by a previous study conducted in Nigeria and Central African Republic, which revealed existence of DDT resistance among Ae. Aegypti (Ayorinde et al., 2015; Ngoagouni et al., 2016).

The present study has found that the mean-time required for knocking down 50\% and 95\% of Ae. Aegypti formosus was lowest and that of $C x$. quinquefasciatus to be highest among the mosquitoes exposed to permethrin (0.75\%) and DDT (4\%). Longer knockdown times (min) are indications of presence of resistance to a particular insecticide, therefore knockdown resistance has been implicated in the $C x$. quinquefasciatus population in this study area. Both Ae. aegypti formosus and An. arabiensis are outdoor biting mosquitoes and might not be controlled by interventions such 
as LLINs and IRs. There is need therefore to opt for other more effective control methods, including larval source management and larviciding for this group of mosquitoes.

The present study has revealed different levels of insecticide susceptibility status to four classes of commonly used insecticides in the most common mosquito vectors of human diseases in north-eastern Tanzania. The study has also provided baseline information on the insecticides susceptibility status of non-malaria mosquito vectors and the current susceptibility status on malaria vectors in the study area. The findings of the present study call for integrated vector control interventions.

\section{Acknowledgements}

The authors acknowledge the financial support from the National Institute for Medical Research through TRAction project. The Kilimanjaro Christian Medical University College is thanked for laboratory analysis of samples. Collins Mweresa, Bruno Mmbando, Heri Bakari, Michael Makange, Joseph Mugasa, Anna Makange and Rukia Ahmed are acknowledged for their support during the implementation of this work. We are grateful for the cooperation given to us by the Muheza community.

\section{References}

Aïkpon, R., Fiacre, A., Razaki. O., Olivier, O., Nazaire, A., Frédéric, O. \& Martin, A. (2013) Bendiocarb resistance in Anopheles gambiae s.l. populations from Atacora. Parasites \& Vectors 6:192.

Ansaria, M. A., Mittal, P. K., Razdan, R. K., Dhiman, R. C. \& Kumar, A. (2004) Evaluation of pirimiphosmethyl ( $50 \%$ EC) against the immatures of Anopheles stephensi/An. culicifacies (malaria vectors) and Culex quinquefasciatus (vector of Bancroftian filariasis). Journal of Vector Borne Diseases 41: $10-16$.

Ayorinde, A., Oboh, B., Oduola, A. \& Otubanjo, O. (2015) The insecticide susceptibility status of Aedes Aegypti (Diptera : Culicidae ) in farm and nonfarm sites of Lagos State, Nigeria. Journal of Insect Sciences 15: 2-5.

Bass, C., Nikou, D., Donnelly, MJ., Williamson, MS., Ranson, H., Ball, A., Vontas, J. \& Field, LM. (2007) Detection of knockdown resistance ( $\mathrm{kdr}$ ) mutations in Anopheles existing methods. Malaria Journal 6: 1-14.

Betson, M., Musa, J. \& Awolola, T.S. (2013) Status of insecticide susceptibility in Anopheles gambiae s.l. from Malaria Surveillance Sites in The Gambia. Malaria Journal 8: 187.

Bracco, J. E., Barata, J. M. \& Marinotti, O. (1999) Evaluation of Insecticide Resistance and biochemical mechanisms in a population of Culex quinquefasciatus (Diptera: Culicidae) from São Paulo, Brazil. Memórias Do Instituto Oswaldo Cruz 94: 115-120.

Chanda, E., Chanda, J., Kandyata, A., Phiri, F.N., Muzia, L., Haque, U. \& Baboo, K.S. (2013) Efficacy of ACTELLIC $300 \mathrm{CS}$, pirimiphos methyl, for indoor residual spraying in areas of high vector resistance to pyrethroids and carbamates in Zambia . Journal of Medical Entomology 50: 12751281.

Chareonviriyaphap, T., Bangs, M.J., Suwonkerd, W., Kongmee, M., Corbel, V \& Ngoen-Klan, R. (2013) Review of insecticide resistance and behavioral avoidance of vectors of human diseases in Thailand. Parasites \& Vectors 6:280.

Choi, K.S., Riann, C., Nardini, L., Wood, O.R., Agubuzo, E., Muleba, M. \& Munyati, S. (2014) Insecticide resistance and role in malaria transmission of Anopheles funestus populations from Zambia and Zimbabwe. Parasites \& Vectors 7: 464.

David, J., Ismail, H.M., Chandor-proust, A. \&, Ingraham, M.J. (2013) Role of cytochrome P450s in 
insecticide resistance: impact on the control of mosquito-borne diseases and use of insecticides on earth. Philosophical Transactions of the Royal Society B: Biological Sciences 368:1612.

Derua, Y.A., Alifrangis, M., Magesa, S.M., Kisinza, W.N. \& Simonsen, P.E. (2015) Sibling species of the Anopheles funestus Group and their infection with malaria and lymphatic filarial parasites, in archived and newly collected specimens from northeastern Tanzania. Malaria Journal 14: 104.

Edwards, F.W. (1941) Mosquitoes of the Ethiopian Region, III Culicine Adults and Pupae. The Oxford University Press, London and Dorking, England.

Gatton, M.L., Chitnis, N., Churcher, T., Donnelly, M.J., Ghani, A.C., Godfray, H.C.J., Gould, F., Hastings, I., Marshall, J., Ranson, H., Rowland, M., Shaman, S.W. \& Lindsay, J. (2013) The Importance of mosquito behavioural adaptations to malaria control in Africa. Evolution 67: 1218-1230.

Gillies, M.T. \& de Meillon, B. (1968) The Anophelinae of Africa South of the Sahara; Ethiopian Zoogeographical Region. 2nd ed. Institute for Medical Research, Johannesburg, South Africa.

Goddard, J. (2008) Dynamics of Arthropod-Borne Diseases. In: Infectious Diseases and Arthropods. Humana Press.

González, T., Bisset, J.A., Díaz, C., Rodríguez, M.M. \& Brandolini, M.B. (1999) Insecticide Resistance in a Culex quinquefasciatus Strain from Rio de Janeiro, Brazil. Memórias Do Instituto Oswaldo Cruz 94: 121-22.

Haji, K.A., Narjis, G., Thawer, B.O. K., Juma H., Mcha, A.R., Abdullah, S. A. \& Christopher, J. (2015) Efficacy, persistence and vector susceptibility to pirimiphos-methyl (Actellic ${ }^{\circledR} 300 \mathrm{CS}$ ) insecticide for indoor residual spraying in Zanzibar. Parasites \& Vectors 8: 628.

Hargreaves, K., Koekemoer, L. L., Brooke, B. D., Hunt, R. H., Mthembu, J. \&Coetzee, M. (2000) Anopheles funestus resistant to pyrethroid insecticides in South Africa. Medical and Veterinary Entomology 14: 181-189.

Huang, Y. (2004) The Subgenus Stegomyia of Aedes in the Afrotropical Region with Keys to the Species (Diptera: Culicidae). Auckland, New Zealand: Magnolia Press.

Jones, C.M., Machin, C., Mohammed, K., Majambere, S., Ali, A.S., Khatib, B.O., Mcha, J., Ranson, H. \& Kelly-Hope, L.A. (2012) Insecticide resistance in Culex quinquefasciatus from Zanzibar: implications for vector control programmes. Parasites \& Vectors 5: 78.

Kabula, B., Tungu, P., Malima, R., Rowland, M., Minja, J., Wililo, R. \& Ramsan, M. (2014) Distribution and spread of pyrethroid and DDT resistance among the Anopheles gambiae complex in Tanzania. Medical and Veterinary Entomology 28: 244-252.

Kabula, B., Tungu, P., Matowo, J., Kitau, J., Mweya, C., Emidi, B., Magesa, S. \& Kisinza, W. (2012) Susceptibility status of malaria vectors to insecticides commonly used for malaria control in Tanzania. Tropical Medicine \& International Health 17: 742-750.

Karlekar, S.R., Deshpande, M.M., \& Andrew, R.J. (2013) Present susceptibility status of Culex quinquefasciatus, Say to three insecticides in Nagpur District of India. Indian Journal of Science and Technology 1 (2): 12-14.

Kisinza, W., Tungu, P., Malima, R., Mbilu, T., Emidi, B., Batengana, B. \& Materu, G. (2013) Malaria Entomological Surveillance in Tanzania Mainland: Detection and Monitoring of Insecticide Resistance to Malaria Vectors. Technical Report of the National Institute for Medical Research, Tanzania.

Macoris, M.L., Andrighetti, M.T.M., Wanderley, D.M.V. \& Ribolla, P.E.M. (2014) Impact of insecticide resistance on the field control of Aedes aegypti in the State of São Paulo. Revista Da Sociedade Brasileira de Medicina Tropical 47 573-578.

Lima, E. P., Paiva, M. H. S., de Araújo, A. P., da Silva, E. V. G., da Silva, U. M., de Oliveira, L. N., Santana, A. E. G., Barbosa, C. N., de Paiva Neto, C. C., Goulart, M. O. F., Wilding, C. S., Ayres, C. F. J. \& de Melo Santos, M. A. V (2011) Insecticide resistance in Aedes aegypti populations from Ceará, Brazil. Parasites \& vectors 4(1):5 
Lwetoijera, D. W., Harris, C., Kiware, S.S., Dongus, S., Devine, G.J., Philip J McCall, P. \& Majambere, S. (2014) Increasing role of Anopheles funestus and Anopheles arabiensis in malaria transmission in the Kilombero Valley, Tanzania. Malaria Journal 13: 331.

Matowo, J., Jones, C., Kabula, B., Ranson, H., Steen, K., Mosha, F.,Rowland, M. \& Weetman, D. (2014) Genetic basis of pyrethroid resistance in a population of Anopheles arabiensis, the primary malaria vector in Lower Moshi, North-Eastern Tanzania. Parasites \& Vectors 7:274.

Matowo, J., Kitau, J., Kaaya, R., Kavishe, R., Wright, A., Kisinza, W. \& Kleinschmidt, I. (2015) Trends in the selection of insecticide resistance in Anopheles gambiae s.l. mosquitoes in northwest Tanzania during a community randomized trial of longlasting insecticidal nets and indoor residual. Medical and Veterinary Entomology 29: 51-59.

Mtove, G., Mugasa, J.P., Messenger, L.A., Malima, R.C., Mangesho, P., Magogo, F., Plucinski, M., Hashimu, R., Matowo, J., Shepard, D., Batengana, B., Cook, J., Emidi, B., Halasa, Y., Kaaya, R., Kihombo, A., Lindblade, K.A., Makenga, G., Mpangala, R., Mwambuli, A., Mzava, R., Mziray, A., Olang, G., Oxborough, R.M., Seif, M., Sambu, E., Samuels, A., Sudi, W., Thomas, J., Weston, S., Alilio, M., Binkin, N., Gimnig, J., Kleinschmidt, I., McElroy, P., Mouton, L.H., Norris, L., Ruebush, T., Venkatesan, M., Rowland, M., Mosha, F.W. \& Kisinza, W.N. (2016) The effectiveness of nonpyrethroid insecticide-treated durable wall lining to control malaria in rural Tanzania: study protocol for a two-armed cluster randomized trial. BMC Public Health 16: 633.

Ngoagouni, C., Kamgang, B., Brengues, C., Yahouedo,G., Paupy, G., Nakouné, E., Kazanji, M. \& Chandre, F. (2016) Susceptibility profile and metabolic mechanisms involved in Aedes aegypti and Aedes albopictus resistant to DDT and deltamethrin in the Central African Republic. Parasites \& Vectors 9: 599.

Nkya, T.E., Akhouayri, I., Kisinza, W. \& David, J. (2013) Impact of environment on mosquito response to pyrethroid insecticides: facts, evidences and prospects. Insect Biochemistry and Molecular Biology 43: 407-416

Nkya, T.E., Akhouayri, I., Poupardin, R., Batengana, B., Mosha, F., Magesa, S., Kisinza, W. \& David, J. (2014) Insecticide resistance mechanisms associated with different environments in the malaria vector Anopheles gambiae: a case study in Tanzania. Malaria Journal 13:28.

Philbert, A., Lyantagaye, S.L. \& Gamba N. (2014). A Review of Agricultural Pesticides Use and the Selection for Resistance to Insecticides in Malaria Vectors. Advances in Entomology 2: 120-28.

Protopopoff, N., Matowo, J., Malima, R., Kavishe, R., Kaaya, R., Wright, A., West, P.A., Kleinschmidt, I., Kisinza, W., Mosha, F.W. \& Rowland, M. (2013). High Level of Resistance in the Mosquito Anopheles gambiae to pyrethroid insecticides and reduced susceptibility to bendiocarb in northwestern Tanzania. Malaria Journal 12:149.

Reid, M.C. \& McKenzie, F.E. (2016) The contribution of agricultural insecticide use to increasing insecticide resistance in African malaria vectors. Malaria Journal 15:107.

Riveron, J.M., Chiumia, M., Menze, B.D., Barnes, K.G., Irving, H., Ibrahim, S.S., Weedall, G.D., Mzilahowa,T. \& Wondji, C.S. (2015) Rise of Multiple Insecticide Resistance in Anopheles funestus in Malawi: A Major Concern for Malaria Vector Control. Malaria Journal 14: 344.

Simonsen, P.E., Derua, Y.A., Magesa, S.M., Pedersen, E.M. ,Stensgaard, A., Malecela, M.N. \& Kisinza, W.N. (2014) Lymphatic filariasis control in Tanga Region, Tanzania : status after eight rounds of mass drug administration. Parasites \& Vectors 7:507.

Umar, A., Kabir, B.G.J., Amajoh, C.N., Inyama, P.U., Ordu, D.A., Barde, A.A. \& Misau, A. (2014) Susceptibility Test of Female Anopheles Mosquitoes to Ten Insecticides for Indoor Residual Spraying ( IRS ) Baseline Data Collection in Northeastern Nigeria. Journal of Entomology and Nematology 6 (7): 98-103.

URT (2013) 2012 Population and Housing Census; Population Distribution by Administrative Areas, United Republic of Tanzania. 
WHO (1975) Manual on Practical Entomology in Malaria. World Health Organization, Geneva.

WHO (2013) Test Procedures for Insecticide Resistance Monitoring in Malaria Vector Mosquitoes. World Health Organization. Geneva, Switzerland.

Yadouleton, A.W., Padonou, G., Asidi, A., Moiroux, N., Bio-banganna, S., Corbel, V. \& Raphael, N. (2010) Insecticide resistance status in Anopheles gambiae in Southern Benin. Malaria Journal 9:83. 